Blood Coagul Fibrinolysis. 2013 Apr;24(3):237-42. doi: 10.1097/MBC.0b013e328359f618.

\title{
The association between the 4G/5G polymorphism in the promoter of the plasminogen activator inhibitor-1 gene and extension of postsurgical calf vein thrombosis.
}

\author{
Ferrara F, Meli F, Raimondi F, Montalto S, Cospite V, Novo G, Novo S. \\ Department of Internal Medicine, Cardiovascular and Nephro-urogical Diseases, University Medical Hospital, \\ Palermo, Italy. f_ferrara@virgilio.it
}

\begin{abstract}
The objective of this study was to evaluate whether the presence of a plasminogen activator inhibitor type 1 (PAl-1) promoter polymorphism 4G/5G could significantly influence the proximal extension of vein thrombosis in spite of anticoagulant treatment in patients with calf vein thrombosis (CVT) following orthopaedic, urological and abdominal surgery. We studied 168 patients with CVT, who had undergone orthopaedic, urological and abdominal surgery, subdivided as follows: first, 50 patients with thrombosis progression; second, 118 patients without thrombosis progression. The $4 \mathrm{G} / 5 \mathrm{G}$ polymorphism of the plasminogen activator inhibitor 1 was evaluated in all patients and in 70 healthy matched controls. We also studied PAI-1 activity in plasma. The presence of $4 \mathrm{G} / 5 \mathrm{G}$ genotype was significantly increased in the group of patients with the extension of thrombotic lesions and was associated with an increase in CVT extension risk (odds ratio adjusted for sex 2.692; 95\% confidence interval 1.302-4.702). Moreover, we observed a significant increase of PAl-1 plasma activity in patients with extension of thrombotic lesion vs. patients without extension $(P=0.0001)$. Patients with $4 G / 5 G$ genotype in the promoter of the plasminogen activator inhibitor - 1 gene present a higher risk of extension of thrombotic lesions.
\end{abstract}

